\title{
Carbon utilization phenome of Leptospira interrogans serovar Manilae strain K64
}

\author{
Ailyn G. Manglicmot-Yabes ${ }^{1, \star}$, Sharon Yvette Angelina M. Villanueva ${ }^{2}$ and \\ Nina G. Gloriani ${ }^{3}$
}

${ }^{1}$ Department of Pharmacology and Toxicology, College of Medicine, University of the Philippines Manila, 547 Pedro Gil Street, PO Box 593, Manila 1000, Philippines.

${ }^{2}$ Department of Medical Microbiology, College of Public Health, University of the Philippines Manila, 625 Pedro Gil Street, Manila 1000, Philippines.

${ }^{3}$ Section of Clinical Microbiology, Institute of Pathology, St Luke's Medical Center, E. Rodriquez Sr. Avenue, Quezon City, Philippines.

\section{ABSTRACT}

Background: Leptospirosis, an acute febrile disease caused by the pathogenic species of genus Leptospira, is one of the neglected emerging zoonoses that is of global public health concern. The recent genus-wide sequencing of Leptospira isolates led to the need for better understanding of the complex metabolic mechanisms of this organism. However, majority of the published studies on Leptospira metabolism were still the pioneering works of Baseman and Cox in the 60's and their contemporaries. Knowledge on the carbon sources that supports the growth of a Leptospira species will not only contribute to the limited metabolic studies but will further support the reported genes and metabolic pathways of this organism. Objective: Thus, this study aimed to describe the carbon utilization phenome of Leptospira interrogans serovar Manilae strain K64, one of the dominantly circulating pathogenic Leptospira in the Philippines. Methods: A previously optimized Biolog ${ }^{\mathrm{TM}}$ Gen III sole carbon utilization phenotype microarray assay protocol for leptospires was adapted. Results: L. interrogans serovar Manilae strain K64 showed utilization of 29 carbon sources belonging to sugars and sugar derivatives, amino acids, methyl ester, carboxylic acid and fatty acids. These were $\mathrm{N}$-acetyl- $\beta$-D-mannosamine, $\mathrm{N}$-acetyl-D-galactosamine, $\mathrm{N}$-acetyl neuraminic acid, Dfructose, D-galactose, 3-methyl glucose, D-fucose, L-fucose, L-rhamnose, inosine, D-fructose-6phosphate, D-gluconic, D-glucuronic acid, glucuronamide, D-saccharic acid, D-aspartic acid, Dserine, L-alanine, L-arginine, L-histidine, L-pyroglutamic acid, L-serine, D-lactic acid methyl ester, citric acid, D-malic acid, L-malic acid, alpha ketoglutaric acid, alpha ketobutyric acid, and acetoacetic acid. Discussion and Conclusion: The carbon sources utilized by L. interrogans serovar Manilae strain K64 agreed well with the identified genes and metabolic pathways among Leptospira species. Moreover, these 29 carbon sources have been previously reported to be associated in the biosynthesis of peptidoglycan, lipopolysaccharide, histidine, sulfur, amino acids, and isoleucine and

* Correspondence Ailyn G. Manglicmot-Yabes ${ }^{1}$ Department of Pharmacology and Toxicology, College of Medicine, University of the Philippines Manila, 547 Pedro Gil Street, PO Box 593, Manila 1000, Philippines. amyabes@up.edu.ph Tel: +63285264248

Received: 1 December 2020 Revised: 15 February 2021 Accepted: 30 March 2021 Published: 8 April 2021

doi https://doi.org/10.28916/1smb.5.1.2021.76 in other metabolic pathways such as glycolysis, pentose-phosphate, pyruvate and fatty acid in Leptospira spp.

Keywords: Leptospirosis; Leptospira interrogans; phenotype microarray; carbon utilization phenome

\section{INTRODUCTION}

Leptospirosis is a preventable disease in animals and humans. In majority of the developing countries in the Asia Pacific region and in the Philippines, poor living conditions along with frequent typhoons, being largely a water-borne zoonoses, contribute to the risk of infection (Victoriano et al., 2009). It is now an emerging global disease due to the re-emergence of the disease in non-endemic areas and becoming an urban problem in highly endemic areas (Kariv et al., 2001; Picardeau, 2013). To elucidate the current disease burden of leptospirosis, studies are focused on the need for continued monitoring of the prevailing serovars in a given geographical area and improvement of diagnostic capabilities (Gloriani et al., 2016a; Gloriani et al., 2016b; Saito et al., 2017; Tabo et al., 2018; Villanueva et al., 2010; Villanueva et al., 2014; Villanueva et al., 2016; Villanueva et al., 2018; Yanagihara et al., 2007; Zamora \& Gloriani, 2015). 


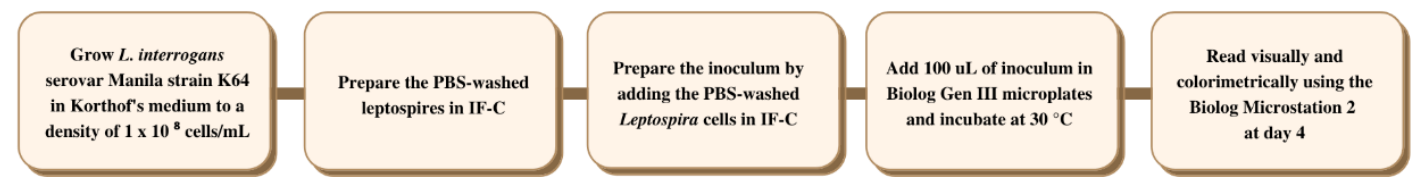

Figure 1: Biolog ${ }^{\mathrm{TM}}$ Gen III sole carbon utilization phenotype microarray assay for Leptospira

The recent genus-wide sequencing of Leptospira isolates further elucidated the biodiversity, epidemiology and evolution of this pathogen (Guglielmini et al., 2019). This, however, led to the need for better understanding of the complex metabolic mechanism in Leptospira species. Based on published studies, the entire metabolic process in Leptospira is not yet clear, even the pentose-phosphate pathway also needs further understanding (Govindaraju et al., 2017). The absence of glycolysis even in the presence of all the genes to produce glycolytic enzymes remains to be further studied (Kefford et al., 1986; Nascimiento et al., 2004; Picardeau et al., 2008; Ren et al., 2003; Zhang et al., 2011). Interestingly, many aspects of Leptospira energy metabolism were understood when the genome of two Leptospira strains (L. interrogans serovar Copenhageni and $L$. interrogans serovar Lai) were completely sequenced (Nascimiento et al., 2004).

To date, majority of the published studies on Leptospira metabolism, particularly on the nutritional requirements, were still the pioneering works of Baseman and Cox in the 60's and their contemporaries (Baseman \& Cox, 1969; Hennerbery \& Cox, 1971). Thus, this study aimed to describe the carbon utilization phenome of Leptospira interrogans serovar Manilae strain K64 using the sole carbon utilization phenotype microarray (PM), known to be the third major technology utilized in the omics-driven research and drug development (Bochner et al., 2001; Bochner, 2003; Bochner, 2009). Knowledge on the carbon utilization phenome of one of the four Leptospira serovar/serogroups that was previously reported to be predominantly circulating in the Philippines, will not only contribute to the limited metabolic studies, but will further support the reported genes and metabolic pathways of this organism, as well as identify potential drug targets. In addition, similar to the previously reported uses of Biolog ${ }^{\mathrm{TM}}$ phenotype microarray technology in the identification of many gram positive and gram negative microorganisms and in mechanism of action studies of novel antimicrobials, its use as a diagnostic tool for leptospirosis by comparing the phenome between pathogenic and nonpathogenic Leptospira serovar, as well as, its use as a screening for and elucidating the mechanism/s of potential antimicrobials for leptospirosis could be the future directions of this study.

\section{MATERIALS AND METHODS Equipment and reagents}

The Biolog ${ }^{\mathrm{TM}}$ Gen III MicroStation System (Biolog ${ }^{\mathrm{TM}}$ Inc., Hayward, CA, USA) was used in the sole carbon source utilization phenotype microarray assay. The Gen III Microplate ${ }^{\mathrm{m}}$ and the inoculating fluid (IFC) used in the sole carbon source utilization assay were all obtained from Biolog $^{\mathrm{TM}}$ (Biolog ${ }^{\mathrm{TM}}$ Inc., Hayward, CA, USA). Phosphate buffered saline (PBS), and Leptospira culture medium (i.e., Korthof's medium) were of analytical grade.

\section{Leptospira strain and Inoculum preparation}

Stock culture of L. interrogans serovar Manilae strain K64 was obtained from the Leptospirosis Prevention and Control Laboratory (LepCon), Department of Medical Microbiology, College of Public Health, University of the Philippines Manila. The organism was maintained by continuous culture in Korthof's medium. The inoculum (PBS-washed Leptospira cells in IF-C), was prepared using L. interrogans serovar Manilae strain K64 grown in Korthof's at $30^{\circ} \mathrm{C}$ for 4-7 days with bacterial density of approximately $1 \times 10^{8}$ cells $/ \mathrm{mL}$, as previously described (Manglicmot-Yabes et al., 2020). Biolog $^{\mathrm{TM}}$ phenotype microarray (PM) sole carbon utilization
technology

The Biolog ${ }^{\mathrm{TM}}$ Gen III microplate was used to analyze the ability of L. interrogans serovar Manilae strain K64 to metabolize 71 major classes of biochemicals. These biochemicals, pre-coated onto the 96 microplate, include carbon sources belonging to sugars and sugar derivatives $(\mathrm{n}=35)$, amino acids $(\mathrm{n}=11)$, and esters, carboxylic acids and fatty acids $(\mathrm{n}=25)$. A previously described protocol in another study was adapted. The method was a modification of the Biolog ${ }^{\mathrm{TM}}$ Gen III sole carbon utilization phenotype microarray standard protocol, optimized for its suitability to evaluate the anti-leptospiral activity of a plant extract and selected antimicrobials against Leptospira serovars, was adapted (Manglicmot-Yabes et al., 2020). Briefly, $100 \mathrm{uL}$ of inoculum (PBSwashed L. interrogans serovar Manilae strain K64 cells in IF-C), was aseptically dispensed to each well of the Biolog ${ }^{\mathrm{TM}}$ Gen III microplates and incubated at $30^{\circ} \mathrm{C}$ (Figure 1). The procedure was performed in 2 runs in triplicates. Only three replicates per run were used since the manufacturer's claim for reproducibility in the package insert is excellent due to controlled conditions required in the whole procedure and on the basis of the consistent results obtained in the previous study (Manglicmot-Yabes et al., 2020). Those wells with corrected OD of $>0.100$ and visually comparable with the positive control were considered well utilized and interpreted as positive for carbon source utilization. On the other hand, those wells with corrected ODs of $<0.100$, although this is above the corrected OD of the negative control, were considered negative or borderline and interpreted as negative for carbon source utilization.

\section{Ethical approvals}

This research was registered in the University of the PhilippinesManila (UP) Research Grant and Administration Office (RGAO 20191090) and underwent the Institutional Biosafety and Biosecurity Committee (IBBC 2019-014), and Research Ethics Review Board (2020089-EX) reviews and approvals. All experiments using Leptospira cultures were performed in a university-based BSL-II facility.

\section{RESULTS}

Twenty nine carbon sources were utilized by L. interrogans serovar Manilae strain K64 out of the 71 major classes of biochemicals precoated in Biolog ${ }^{\mathrm{m}}$ Gen III microplate (Table 1). These carbon sources belong to sugars and sugar derivatives $(n=15)$, amino acids $(n=7)$, and carbon sources which belong to esters, carboxylic acids, and fatty acids $(n=7)$. Results of the carbon utilization phenome or pattern of carbon utilization of $L$. interrogans serovar Manilae strain K64 were consistent within the 3 replicates and between two runs.

\section{DISCUSSION}

The carbon sources reported to be utilized by L. interrogans serovar Manilae strain K64 in this study had been previously reported in other Leptospira species to be associated in biosynthesis of peptidoglycan, lipopolysaccharide, histidine, sulfur, amino acids, isoleucine and other metabolic pathways such as glycolysis, pentose-phosphate, pyruvate and fatty acid (Amineni et al., 2010; Charon et al., 1973; Gerhardt \& Ball 1959; Govindaraju, 2017; Nascimento et al., 2004; Ren, 2003; Ricaldi et al., 2012; Vin et al., 1985; Zhang et al., 2011). 
Table 1: Carbon sources utilized by L. interrogans serovar Manilae strain K64 using the Biolog ${ }^{\text {TM }}$ Gen III phenotype microarray

\begin{tabular}{|c|c|c|}
\hline Sugars and sugar derivatives $\left(n=15 / 35^{\star}\right)$ & $\begin{array}{l}\text { Amino Acids } \\
\left(\mathrm{n}=7 / 11^{\star}\right)\end{array}$ & $\begin{array}{l}\text { Esters, carboxylic acids and fatty acids } \\
\left(\mathrm{n}=7 / 25^{\star}\right)\end{array}$ \\
\hline 1. $\mathrm{N}$-acetyl- $\beta$-D-mannosamine & 1. D-aspartic acid & 1. D-lactic acid methyl ester \\
\hline 2. $\mathrm{N}$-acetyl-D-galactosamine & 2. D-serine & Citric acid \\
\hline 3. N-acetyl neuraminic acid & 3. L-alanine & D-malic acid \\
\hline 4. D-fructose & 4. L-arginine & 4. L-malic acid \\
\hline 5. D-galactose & 5. L-histidine & 5. Alpha ketoglutaric acid \\
\hline 6. 3-methyl glucose & 6. L-pyroglutamic acid & Alpha ketobutyric acid \\
\hline 7. D-fucose & 7. L-serine & Acetoacetic acid \\
\hline 8. L-fucose & & \\
\hline 9. L-rhamnose & & \\
\hline 10. Inosine & & \\
\hline 11. D-fructose-6-phosphate & & \\
\hline 12. D-Gluconic & & \\
\hline 13. D-glucuronic acid & & \\
\hline 14. Glucuronamide & & \\
\hline 15. D-saccharic acid & & \\
\hline
\end{tabular}

*Total number of carbon source belonging to the group

L. interrogans serovar Manilae strain K64 utilized amino sugars believed to be associated with lipopolysaccharides and peptidoglycan synthesis. These were N-acetyl- $\beta$-D-mannosamine, N-acetyl-Dgalactosamine and $\mathrm{N}$-acetyl neuraminic acid. In a study using gas liquid chromatography analysis, N-acetyl-D-galactosamine was also found in leptospiral lipopolysaccharide of $L$. interrogans serovar copenhageni strain L45 (Vin et al., 1985). Moreover, N-acetylneuraminic acid biosynthetic pathway was also reported in L. interrogans serovar Copenhageni strain L1-130 (Ricaldi et al., 2012). This was consistent with the finding of the present study wherein L. interrogans serovar Manilae strain K64 utilized N-acetyl neuraminic acid. Other sugar and sugar derivatives such as the D-galactose, 3-methyl glucose, D-fucose, Lfucose, D-fructose, L-fucose, inosine, D-fructose-6-phosphate, Dgluconic acid, D-glucuronic acid, glucuronamide, and saccharic acid were also utilized by L. interrogans serovar Manilae strain K64. The utilization of these carbon sources was consistent with the previous reports that Leptospira has a functional glycolytic metabolism pathway. In the study of Nascimento et al. (2004), the gene LA_1437 of $L$. interrogans serogroup Icterohaemorrhagiae serovar Lai strain 56601 was reported to be potentially encoding a glucokinase (GLK). Similarly, a gene which is potentially encoding GLK was also reported in L. biflexa strain Patoc 1 (Kefford et al., 1986; Picardeau et al., 2013).

Amino acid biosynthesis have been reported in Leptospira interrogans serovars Semaranga Tarassovi, and Canicola using radioactive carbon dioxide on growing cells and paper chromatography (Charon et al., 1973; Gerhardt \& Ball 1959). Similarly, the need for amino acids to support the growth of $L$. interrogans serovar Manilae strain K64 was also exhibited as evidenced by the utilization of seven amino acids such as D-aspartic acid, D-serine, L-serine, L-alanine, Larginine, L-histidine, and L-pyroglutamic acid.

$\beta$-oxidation of fatty acids was thought to be the only source of energy and carbon in Leptospira as reported in the enzymatic analysis of cell-free extracts of the three Leptospira strains (Baseman \& Cox, 1969; Henneberry \& Cox, 1971). Thus, the long chain fatty acids, Tween 80 (polyoxyethylene sorbitan monooleate), Tween 60 (polyoxyethylene sorbitan monostearate) and Tween 40 (polyoxyethylene sorbitan monopalmitate) were usually employed as carbon source in the metabolism of Leptospira. Unfortunately, the Biolog ${ }^{\mathrm{TM}}$ Gen III panel only includes Tween 40, which apparently, was poorly utilized by $L$. interrogans serovar Manilae strain K64. This result was consistent with the early studies of Cox and Baseman (1969), in which Tween 40 was also unexpectedly, poorly utilized by a $\mathrm{B}_{16}$ water strain of Leptospira. To fully elucidate the fatty acid utilization of $L$. interrogans serovar Manilae strain K64, other Biolog ${ }^{\mathrm{TM}}$ PM panel, which contain more comprehensive fatty acid substrates is highly recommended in future studies. Although the current panel used in this initial study has limited fatty acid substrates, it already screened for 71 carbon sources which are necessary for the growth of mostly gram negative and gram positive bacteria (Bochner et al., 2001; Bochner, 2003; Bochner, 2009).

\section{CONCLUSION}

To the knowledge of the authors, this is the first study reporting the carbon utilization phenome of a Leptospira strain using the BiologTM sole carbon source utilization phenotype microarray technology. The carbon sources that favor the growth of L. interrogans serovar Manilae strain K64 obtained in this study were consistent with the reported genes and metabolic pathways among Leptospira species. Moreover, these carbon sources utilized by L. interrogans serovar Manilae strain K64 have been previously reported to be associated in the biosynthesis of peptidoglycan, lipopolysaccharide, histidine, sulfur, amino acids, isoleucine and in other metabolic pathways such as glycolysis, pentosephosphate, pyruvate and fatty acid in Leptospira spp.

\section{DISCLOSURES}

This paper was presented during the $32^{\text {nd }}$ Faculty Research Forum in the University of the Philippines College of Medicine as a poster presentation. The authors declare no conflicts of interest in this work and the manuscript was approved by all the authors, as well as the funding organization, for publication.

\section{ACKNOWLEDGEMENT}

The authors extend gratitude to the Department of Medical Microbiology, College of Public Health, University of the PhilippinesManila for providing the Leptospira strain and for allowing the researchers to conduct all the experiments in its BSL-2 facility. The authors also acknowledge the National Institutes of Health at the University of the Philippines for the research grant of the main study (NIH-2019-006), in which this paper is one of its outputs. Lastly, the authors are also grateful to the project assistants, Monica Angelique O. Ramos and Katelyn H. Grace De Jesus, for the assistance in the laboratory work and to Prof. Dr. Chea Yoke Kqueen of Universiti of Putra Malaysia, Malaysia for his expert opinion on the use of the Biolog phenotype microarray.

\section{REFERENCES}

Baseman, J.B., \& Cox, C. D. (1969). Intermediate energy metabolism of Leptospira. Journal of bacteriology, 97(3), 992-1000. https://doi.org/10.1128/JB.97.3.992-1000.1969

Bochner, B. R., Gadzinski, P., \& Panomitros, E. (2001). Phenotype microarrays for high-throughput phenotypic testing and assay of gene function. Genome 
Research, 11(7), 1246-1255.

https://doi.org/10.1101/gr.186501

Bochner, B. R. (2003). New technologies to assess genotype-phenotype relationships. Nature Reviews Genetics, 4(4), 309-314. https://doi.org/10.1038/nrg1046

Bochner B. R. (2009). Global phenotypic characterization of bacteria. FEMS Microbiology Reviews, 33(1), 191-205. https://doi.org/10.1111/j.1574-6976.2008.00149.x

Charon, N. W., Johnson, R. C., \& Peterson, D. (1973). Amino acid biosynthesis in the spirochete Leptospira: evidence for a novel pathway of isoleucine biosynthesis. Journal of Bacteriology, 117(1), 203-211. https://doi.org/10.1128/JB.117.1.203-211.1974

Gerhardt, M. R., \& Ball, M. G. (1959). Amino acid utilization by Leptospira canicola. Journal of bacteriology, 77(1), 17-22. https://doi.org/10.1128/JB.77.1.17-22.1959

Gloriani, N. G., Villanueva, S. Y. A. M., Yanagihara, Y., \& Yoshida, S. I. (2016). Post-Flooding Surveillance of Leptospirosis After the Onslaught of Typhoons Nesat, Nalgae And Washi in the Philippines. Southeast Asian J Trop Med Public Health, 47(4), 774-786.

Gloriani, N. G., Villanueva, S. Y. A. M., Yanagihara, Y., \& Yoshida, S. I. (2016). Identification of prevalent Leptospira serovars infecting water buffaloes, cows, dogs, pigs, and rats in the Philippines. Southeast Asian Journal of Tropical Medicine in Public Health, 47(4), 766-773.

Govindaraju, S., Vinayagamurthy B., Swamy, N., \& Shivamallu, N. (2017). Fatty Acid Metabolism in Leptospira a Key to its Pathogenicity and Evasion from Host Immune Response Leading to Prolonged Survival of the Organism. Indian Journal of Natural Sciences. 7(40):11939-11945.

Guglielmini, J., Bourhy, P., Schiettekatte, O., Zinini, F., Brisse, S., \& Picardeau, M. (2019). Genus-wide Leptospira core genome multilocus sequence typing for strain taxonomy and global surveillance. PLoS neglected tropical diseases, 13(4), e 0007374 https://doi.org/10.1371/journal.pntd.0007374

Henneberry, R. C. Cox, C. D. (1971). Beta-oxidation of fatty acids by Leptospira. Can J Microbiol, 16(1), 41-45. https://doi.org/10.1139/m70-007

Kariv, R., Klempfner, R., Barnea, A., Sidi, Y., \& Schwartz, E. (2001). The Changing Epidemiology of Leptospirosis in Israel. Emerging Infectious Diseases, 7(6), 990-992. https://doi.org/10.3201/eid0706.010611

Kefford, B., Humphrey, B. A., \& Marshall, K. C. (1986). Adhesion: a possible survival strategy for leptospires under starvation conditions. Current Microbiology, 13(5), 247-250. https://doi.org/10.1007/BF01568647

Manglicmot-Yabes A., Villanueva, S.Y.A.M. \& Gloriani, N. (2020). Optimized tube dilution technique and sole carbon utilization assay for anti-leptospiral in vitro screening of plant extracts. Pediatric Infectious Disease Society of the Philippines Journal. 21(2), 3-12.

Nascimento, A. L. T. O. Ko, A. I.Martins, E. A. L.Monteiro-Vitorello, C. B.Ho, P. L.Haake, D. J. C.Van Sluys, M. A. (2004). Comparative Genomics of Two Leptospira interrogans Serovars Reveals Novel Insights into Physiology and Pathogenesis. Journal of Bacteriology, 186(7), 2164-2172. https://doi.org/10.1128/JB.186.7.2164-2172.2004

Picardeau, M. (2013). Diagnosis and epidemiology of leptospirosis. Médecine et maladies infectieuses, 43(1), 1-9. https://doi.org/10.1016/j.medmal.2012.11.005

Picardeau, M., Bulach, D. M., Bouchier, C., Zuerner, R. L., Zidane, N., Wilson, P. J., \& McGrath, A. (2008). Genome sequence of the saprophyte Leptospira biflexa provides insights into the evolution of Leptospira and the pathogenesis of leptospirosis. PlOS One, 3(2). https://doi.org/10.1371/journal.pone.0001607

Ren, S. X., Fu, G., Jiang, X. G., Zeng, R., Miao, Y. G., Xu, H., \& Jiang, H. Q. (2003). Unique physiological and pathogenic features of Leptospira interrogans revealed by whole-genome sequencing. Nature, 422(6934), 888-893. https://doi.org/10.1038/nature01597

Ricaldi, J. N., Matthias, M. A., Vinetz, J. M., \& Lewis, A. L. (2012). Expression of sialic acids and other nonulosonic acids in Leptospira. BMC Microbiology, 12,161 . https://doi.org/10.1186/1471-2180-12-161

Saito, M., Nikaido, Y., Matsumoto, M., Ogawa, M., \& Villanueva, S. Y. A. M. (2017). The current status of diagnostic tools for leptospirosis. Rinsho Biseibutsu Jinsoku Shindan Kenkyukai shi= JARMAM: Journal of Reviews, 33(1), 191-205.

Tabo, N. A., Villanueva, S. Y. A. M., \& Gloriani, N. G. (2018). Prevalence of Leptospira-agglutinating Antibodies in Abattoir Workers and Slaughtered Animals in Selected Slaughterhouses in Cavite, Philippines. Philippine Journal of Science, 147(1), 27-35.

Victoriano, A. F. B., Smythe, L. D., Gloriani-Barzaga, N., Cavinta, L. L., Kasai, T., Limpakarnjanarat, K., \& Yanagihara, Y. (2009). Leptospirosis in the Asia
Pacific region. BMC infectious diseases, 9(1), 147

https://doi.org/10.1186/1471-2334-9-147

Villanueva, M. A., Mingala, C. N., Gloriani, N. G., Yanagihara, Y., Isoda, N., Nakajima, C., \& Koizumi, N. (2016). Serological investigation of Leptospira infection and its circulation in one intensive-type water buffalo farm in the Philippines. Japanese Journal of Veterinary Research, 64(1), 15-24.

Villanueva, S. Y. A. M., Baterna, R. A., Cavinta, L. L., Yanagihara, Y., Gloriani, N. G., \& Yoshida, S. I. (2018). Seroprevalence of Leptospirosis among Water Buffaloes, Pigs, and dogs in Selected Areas in the Philippines, 2007 to 2008 Acta Medica Philippina, 52(1), 110 https://doi.org/10.47895/amp.v52i1.493

Villanueva, S. Y. A. M., Saito, M., Baterna, R. A., Estrada, C. A. M., Rivera, A. K. B., Dato, M. C., \& Masuzawa, T. (2014). Leptospira-rat-human relationship in Luzon, Philippines. Microbes and infection, 16(11), 902-910. https://doi.org/10.1016/j.micinf.2014.07.001

Villanueva, S. Y., Ezoe, H., Baterna, R. A., Yanagihara, Y., Muto, M., Koizumi, N., \& Gloriani, N. G. (2010). Serologic and molecular studies of Leptospira and leptospirosis among rats in the Philippines. The American journal of tropical medicine and hygiene, 82(5), 889-898. https://doi.org/10.4269/ajtmh.2010.09-0711

Vin, T., Adler, B., \& Faine, S. (1985). Ultrastructure and chemical composition of lipopolysaccharide extracted from Leptospira interrogans serovar copenhageni. Microbiology, 132(1), 103-109. https://doi.org/10.1099/00221287-132-1-103

Yanagihara, Y., Villanueva, S. Y., Yoshida, S. I., Okamoto, Y., \& Masuzawa, T. (2007). Current status of leptospirosis in Japan and Philippines. Comparative immunology, microbiology and infectious diseases, 30(5-6), 399-413. https://doi.org/10.1016/j.cimid.2007.05.003

Zamora, P. R. F. C. \& Gloriani, N. G. (2015). Serologic Characterization of Leptospira among Rats Trapped in Selected Public Markets in Iloilo City, Philippines. Acta Medica Philippina, 49(4), 69-73. https://doi.org/10.47895/amp.v49i4.910

Zhang, Q., Zhang, Y., Zhong, Y., Ma, J., Peng, N., Cao, X., \& Zhao, G. (2011). Leptospira interrogans encodes an ROK family glucokinase involved in a cryptic glucose utilization pathway. Acta Biochimica et Biophysica Sinica, 43(8), 618-629. https://doi.org/10.1093/abbs/gmr049

Citation:

Manglicmot-Yabes, A. G., Villanueva, S. Y. A. M. ., \& Gloriani, N. G. (2021). Carbon utilization phenome of Leptospira interrogans serovar Manilae strain K64. Life Sciences, Medicine and Biomedicine, 5(1). https://doi.org/10.28916/lsmb.5.1.2021.76

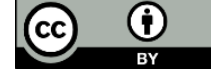

Copyright $\odot 2021$ by the Author(s). Life Sciences, Medicine and Biomedicine (ISSN: 2600-7207) Published by Biome Journals - Biome Scientia Sdn Bhd. Attribution 4.0 International (CC BY 4.0). This open access article is distributed based on the terms and conditions of the Creative Commons Attribution license https://creativecommons.org/licenses/by/4.0/

Life Sciences, Medicine and Biomedicine ISSN: 2600-7207 